\title{
A congruence involving the quotients of Euler and its applications (II)
}

by

\author{
T. X. CAi, X. D. Fu and X. Zhou (Hangzhou)
}

1. Introduction. In [1], the first author showed that for any odd $n>1$,

$$
\sum_{\substack{r=1 \\(r, n)=1}}^{(n-1) / 2} \frac{1}{r} \equiv-2 q_{2}(n)+n q_{2}^{2}(n)\left(\bmod n^{2}\right),
$$

where

$$
q_{i}(n)=\frac{i^{\phi(n)}-1}{n}, \quad(i, n)=1,
$$

is Euler's quotient of $n$ with base $i$. In particular, if $n$ is prime, (1) becomes Lehmer's famous congruence (cf. [2] or [3])

$$
\sum_{r=1}^{(p-1) / 2} \frac{1}{r} \equiv-2 q_{2}(p)+p q_{2}^{2}(p)\left(\bmod p^{2}\right),
$$

which, along with some other congruences, plays an important role in studying the first case of Fermat's last theorem. In this paper, we wish to generalize some other congruences of Lehmer to arbitrary positive integer moduli.

THEOREM 1. If $n$ is odd, then

$$
\begin{aligned}
& \sum_{\substack{r=1 \\
(r, n)=1}}^{\lfloor n / 2\rfloor} \frac{1}{n-2 r} \equiv q_{2}(n)-\frac{1}{2} n q_{2}^{2}(n)\left(\bmod n^{2}\right), \quad(3, n)=1, \\
& \sum_{\substack{r=1 \\
(r, n)=1}}^{\lfloor n / 3\rfloor} \frac{1}{n-3 r} \equiv \frac{1}{2} q_{3}(n)-\frac{1}{4} n q_{3}^{2}(n)\left(\bmod n^{2}\right), \quad(3, n)=1,
\end{aligned}
$$

2000 Mathematics Subject Classification: 11A25, 11B65, $11 \mathrm{~B} 68$.

Key words and phrases: quotients of Euler, Bernoulli numbers, binomial coefficients. Research partly supported by the project NNSFC 10371107. 


$$
\begin{aligned}
& \sum_{\substack{r=1 \\
(r, n)=1}}^{\lfloor n / 4\rfloor} \frac{1}{n-4 r} \equiv \frac{3}{4} q_{2}(n)-\frac{3}{8} n q_{2}^{2}(n)\left(\bmod n^{2}\right), \quad(3, n)=1, \\
& \sum_{\substack{r=1 \\
(r, n)=1}}^{\lfloor n / 6\rfloor} \frac{1}{n-6 r} \equiv \frac{1}{3} q_{2}(n)+\frac{1}{4} q_{3}(n)-\frac{1}{6} n q_{2}^{2}(n)-\frac{1}{8} n q_{3}^{2}(n)\left(\bmod n^{2}\right), \\
& (15, n)=1 .
\end{aligned}
$$

As an application of Theorem 1, we have the following result.

Theorem 2. If $n$ is odd, then

$$
\prod_{d \mid n}\left(\begin{array}{l}
d-1 \\
\lfloor d / 3\rfloor
\end{array}\right)^{\mu(n / d)} \equiv(-1)^{\phi_{3}(n)}\left(3^{\phi(n)+1}-1\right) / 2\left(\bmod n^{2}\right), \quad(3, n)=1,
$$

$$
\prod_{d \mid n}\left(\begin{array}{l}
d-1 \\
\lfloor d / 4\rfloor
\end{array}\right)^{\mu(n / d)} \equiv(-1)^{\phi_{4}(n)}\left(3 \cdot 2^{\phi(n)}-2\right)\left(\bmod n^{2}\right), \quad(3, n)=1,
$$

$$
\begin{aligned}
& \prod_{d \mid n}\left(\begin{array}{l}
d-1 \\
\lfloor d / 6\rfloor
\end{array}\right)^{\mu(n / d)} \equiv(-1)^{\phi_{6}(n)}\left(2^{\phi(n)+2}+3^{\phi(n)+1}-5\right) / 2\left(\bmod n^{2}\right), \\
& (15, n)=1,
\end{aligned}
$$

where

$$
\phi_{e}(n)=\sum_{d \mid n} \mu\left(\frac{n}{d}\right)\left\lfloor\frac{d}{e}\right\rfloor
$$

is the generalized Euler totient function.

REMARK. We shall prove in Lemma 4 below that $(-1)^{\phi_{e}(n)}=-1$ only when $n$ is a prime power $p^{\alpha}$ with $p \equiv 2(\bmod 3)$ for $e=3, p \equiv 5$ or $7(\bmod 8)$ for $e=4$, and $p \equiv 7$ or $11(\bmod 12)$ for $e=6$.

Corollary 1. If $p, q$ are distinct odd primes, then

$$
\begin{aligned}
\left(\begin{array}{l}
p q-1 \\
\lfloor p q / 3\rfloor
\end{array}\right) & \equiv \frac{1}{2}\left(3^{\phi(p q)+1}-1\right)\left(\begin{array}{l}
p-1 \\
\lfloor p / 3\rfloor
\end{array}\right)\left(\begin{array}{l}
q-1 \\
\lfloor q / 3\rfloor
\end{array}\right)\left(\bmod p^{2} q^{2}\right), \\
\left(\begin{array}{l}
p q-1 \\
\lfloor p q / 4\rfloor
\end{array}\right) & \equiv\left(3 \cdot 2^{\phi(p q)}-2\right)\left(\begin{array}{l}
p-1 \\
\lfloor p / 4\rfloor
\end{array}\right)\left(\begin{array}{c}
q-1 \\
\lfloor q / 4\rfloor
\end{array}\right)\left(\bmod p^{2} q^{2}\right), \\
\left(\begin{array}{l}
p q-1 \\
\lfloor p q / 6\rfloor
\end{array}\right) & \equiv \frac{1}{2}\left(2^{\phi(p q)+2}+3^{\phi(p q)+1}-5\right)\left(\begin{array}{l}
p-1 \\
\lfloor p / 6\rfloor
\end{array}\right)\left(\begin{array}{l}
q-1 \\
\lfloor q / 6\rfloor
\end{array}\right)\left(\bmod p^{2} q^{2}\right) .
\end{aligned}
$$

However, we have not been able to generalize the following formula of Lehmer to arbitrary positive integer moduli:

$$
\sum_{r=1}^{\lfloor p / 4\rfloor} \frac{1}{r^{2}} \equiv(-1)^{(p-1) / 2} 4 E_{p-3}(\bmod p)
$$


for any prime $p \geq 5$, where $E_{2 n}$ is the $2 n$th Euler number which can be defined by the generating function

$$
\sec x=\sum_{n=0}^{\infty}(-1)^{n} E_{2 n} \frac{x^{2 n}}{(2 n) !} \quad \text { for }|x|<\frac{\pi}{2} .
$$

What we prove is the following result.

THEOREm 3. If $p$ is an odd prime and $l \geq 1$, then

$$
\sum_{\substack{r=1 \\ p \nmid r}}^{\left\lfloor p^{l} / 4\right\rfloor} \frac{1}{r^{2}} \equiv(-1)^{\left(p^{l}-1\right) / 2} 4 E_{\phi\left(p^{l}\right)-2} \begin{cases}\left(\bmod p^{l}\right) & \text { for } p \geq 5, \\ \left(\bmod 3^{l-1}\right) & \text { for } p=3 .\end{cases}
$$

\section{Auxiliary results}

LEMma 1. If $p \geq 5$ is a prime and $k \geq 2, l, t$ are positive integers, and $s$ is the least positive residue of $p^{l}$ modulo $t$, then

$$
\begin{aligned}
& \text { (9) } \sum_{r=1}^{\left\lfloor p^{l} / t\right\rfloor}\left(p^{l}-t r\right)^{2 k-1} \equiv \frac{t^{2 k-1}}{2 k}\left\{B_{2 k}-B_{2 k}\left(\frac{s}{t}\right)+\frac{p^{2 l}}{t^{2}}\left(\begin{array}{c}
2 k \\
2
\end{array}\right) B_{2 k-2}\right\}\left(\bmod p^{2 l}\right), \\
& \text { (10) } \sum_{r=1}^{\left\lfloor p^{l} / t\right\rfloor}\left(p^{l}-t r\right)^{2 k} \equiv \frac{t^{2 k}}{2 k+1}\left\{\frac{2 k+1}{t} p^{l} B_{2 k}-B_{2 k+1}\left(\frac{s}{t}\right)\right\}\left(\bmod p^{3 l-1}\right), \\
& \frac{p B_{\phi\left(p^{2 l}\right)}}{p-1} \equiv 1\left(\bmod p^{2 l}\right),
\end{aligned}
$$

where $B_{n}$ is the nth Bernoulli number.

Proof. It is well-known that

$$
B_{v+1}(x+1)-B_{v+1}(x)=(v+1) x^{v} .
$$

We let $x=\left(p^{l}-r t\right) / t\left(r=1, \ldots,\left\lfloor p^{l} / t\right\rfloor\right)$, and add all the resulting equations; after cancellation we obtain

$$
\sum_{r=1}^{\left\lfloor p^{l} / t\right\rfloor}\left(p^{l}-t r\right)^{v}=\frac{t^{v}}{v+1}\left\{B_{v+1}\left(\frac{p^{l}}{t}\right)-B_{v+1}\left(\frac{s}{t}\right)\right\},
$$

where we have written $s$ for the least positive residue of $p^{l}$ modulo $t$. From

$$
B_{v}(x)=\sum_{r=0}^{v}\left(\begin{array}{l}
v \\
r
\end{array}\right) B_{r} x^{v-r}
$$


and the von Staudt-Clausen theorem, for $k \geq 2$ we have

$$
\begin{aligned}
B_{2 k}\left(\frac{p^{l}}{t}\right) & \equiv B_{2 k}+\frac{p^{2 l}}{t^{2}}\left(\begin{array}{c}
2 k \\
2
\end{array}\right) B_{2 k-2}\left(\bmod p^{2 l}\right), \\
B_{2 k+1}\left(\frac{p^{l}}{t}\right) & \equiv B_{2 k} \frac{2 k+1}{t} p^{l}\left(\bmod p^{3 l-1}\right) .
\end{aligned}
$$

Taking $v=2 k$ and $v=2 k+1$ in (12), from the above congruences we get (9) and (10) respectively.

Now we prove (11). Taking $2 k=\phi\left(p^{2 l}\right)$ and $t=1$ in (10), noting that $B_{2 k+1}(1)=0$, we have

$$
\sum_{\substack{r=1 \\(r, p)=1}}^{p^{l}-1} r^{\phi\left(p^{2 l}\right)} \equiv \sum_{r=1}^{p^{l}}\left(p^{l}-r\right)^{\phi\left(p^{2 l}\right)} \equiv p^{l} B_{\phi\left(p^{2 l}\right)}\left(\bmod p^{3 l-1}\right) .
$$

By using the fundamental congruence

$$
q_{a}\left(p^{2 l}\right)+q_{b}\left(p^{2 l}\right) \equiv q_{a b}\left(p^{2 l}\right)\left(\bmod p^{2 l}\right)
$$

we have

$$
\begin{aligned}
\sum_{\substack{r=1 \\
(r, p)=1}}^{p^{l}-1} q_{r}\left(p^{2 l}\right) & \equiv \frac{\left(\prod_{1 \leq r \leq p^{l}, p \nmid r} r\right)^{\phi\left(p^{2 l}\right)}-1}{p^{2 l}}=\frac{\left(w_{p^{l}} p^{l}-1\right)^{\phi\left(p^{2 l}\right)}-1}{p^{2 l}} \\
& \equiv-\phi\left(p^{l}\right) w_{p^{l}}\left(\bmod p^{l}\right)
\end{aligned}
$$

where

$$
w_{p^{l}}=\left(\prod_{1 \leq i \leq p^{l}, p \nmid i} i+1\right) / p^{l}
$$

is the generalized Wilson quotient. Note that

$$
\sum_{\substack{r=1 \\(r, p)=1}}^{p^{l}-1} r^{\phi\left(p^{2 l}\right)}=\sum_{\substack{r=1 \\(r, p)=1}}^{p^{l}-1}\left(1+q_{r}\left(p^{2 l}\right) p^{2 l}\right) .
$$

Combining (13)-(15), we have

$$
p^{l} B_{\phi\left(p^{2 l}\right)} \equiv \sum_{\substack{r=1 \\(r, p)=1}}^{p^{l}-1} 1=\phi\left(p^{l}\right)\left(\bmod p^{3 l-1}\right),
$$

and (11) is obtained.

Lemma 2 ([2]). If $v$ is even, $e=2,3,4$, or 6 , then

$$
B_{v}\left(\frac{1}{e}\right)=B_{v}\left(\frac{e-1}{e}\right)=\prod_{q \mid e}\left(1-q^{v-1}\right) \frac{B_{v}}{\phi\left(e^{v}\right)},
$$


where $q$ is a prime factor of $e$. If $v$ is odd and $e=4$, then

$$
B_{v}\left(\frac{1}{4}\right)=-B_{v}\left(\frac{3}{4}\right)=-\frac{v E_{v-1}}{4^{v}} .
$$

Lemma 3. If $p \geq 5$ is a prime, $l, d \geq 1, e \geq 2, p \nmid e, d \equiv \pm 1(\bmod e)$, then

$$
\sum_{\substack{r=1 \\ p \nmid r}}^{\left\lfloor d p^{l} / e\right\rfloor} \frac{1}{d p^{l}-e r} \equiv \sum_{\substack{r=1 \\ p \nmid r}}^{\left\lfloor p^{l} / e\right\rfloor} \frac{1}{p^{l}-e r}\left(\bmod p^{2 l}\right) .
$$

Proof. Let

$$
D=\sum_{\substack{r=1 \\ p \nmid r}}^{\left\lfloor d p^{l} / e\right\rfloor} \frac{1}{d p^{l}-e r}-\sum_{\substack{r=1 \\ p \nmid r}}^{\left\lfloor p^{l} / e\right\rfloor} \frac{1}{p^{l}-e r} .
$$

By expanding the summands as geometric series, we have

$$
\begin{aligned}
D & =\sum_{\substack{r=1 \\
p \nmid r}}^{\left\lfloor d p^{l} / e\right\rfloor} \frac{1}{d p^{l}-e r}-\sum_{\substack{r=1 \\
p \nmid r}}^{\left\lfloor d p^{l} / e\right\rfloor} \frac{1}{p^{l}-e r}+\sum_{\substack{r=1 \\
p \nmid r}}^{\left\lfloor d p^{l} / e\right\rfloor} \frac{1}{p^{l}-e r}-\sum_{\substack{r=1 \\
p \nmid r}}^{\left\lfloor p^{l} / e\right\rfloor} \frac{1}{p^{l}-e r} \\
& \equiv-\frac{(d-1) p^{l}}{e^{2}} \sum_{\substack{r=1 \\
p \nmid r}}^{\left\lfloor d p^{l} / e\right\rfloor} \frac{1}{r^{2}}+\sum_{\substack{r=\left[p^{l} / e\right]+1 \\
p \nmid r}}^{\left\lfloor d p^{l} / e\right\rfloor} \frac{1}{p^{l}-e r} \\
& \equiv-\frac{(d-1) p^{l}}{e^{2}} \sum_{\substack{r=1 \\
p \nmid r}}^{\left\lfloor d p^{l} / e\right\rfloor} \frac{1}{r^{2}}-\frac{p^{l}}{e^{2}} \sum_{\substack{r=\left[p^{l} / e\right]+1 \\
p \nmid r}}^{\left\lfloor d p^{l} / e\right\rfloor} \frac{1}{r^{2}}-\frac{1}{e} \sum_{\substack{r=\left[p^{l} / e\right]+1 \\
p \nmid r}}^{\left\lfloor d p^{l} / e\right\rfloor} \frac{1}{r}\left(\bmod p^{2 l}\right) .
\end{aligned}
$$

For any prime $p \geq 5$, let $t$ be the least positive residue of $d$ modulo $e ; t=1$ or $e-1$. Noting that $p \nmid e$ implies $p^{l}-\left(\left\lfloor(e-1) p^{l} / e\right\rfloor+1\right)=\left\lfloor p^{l} / e\right\rfloor$, we derive that

$$
\begin{aligned}
D \equiv & -\frac{(d-1) p^{l}}{e^{2}} \sum_{\substack{r=1 \\
p \nmid r}}^{\left\lfloor t p^{l} / e\right\rfloor} \frac{1}{r^{2}}-\frac{p^{l}}{e^{2}} \sum_{\substack{r=\left\lfloor p^{l} / e\right\rfloor+1 \\
p \nmid r}}^{\left\lfloor t p^{l} / e\right\rfloor} \frac{1}{r^{2}} \\
& -\frac{1}{e}\left(\sum_{\substack{r=\left\lfloor p^{l} / e\right\rfloor+1 \\
p \nmid r}}^{p^{l}} \frac{1}{r}+\sum_{\substack{r=1 \\
p \nmid r}}^{\left\lfloor t p^{l} / e\right\rfloor} \frac{1}{r+d_{0} p^{l}}\right)\left(\bmod p^{2 l}\right),
\end{aligned}
$$

where $d_{0}=(d-t) / e$. Recalling the well-known congruences 
if $t=1$ we get

$$
\sum_{\substack{r=1 \\ p \nmid r}}^{p^{l}} \frac{1}{r} \equiv 0\left(\bmod p^{2 l}\right), \quad \sum_{\substack{r=1 \\ p \nmid r}}^{p^{l}} \frac{1}{r^{2}} \equiv 0\left(\bmod p^{l}\right),
$$

$$
D \equiv\left\{-\frac{(d-1)}{e^{2}}+\frac{d_{0}}{e}\right\} p^{l} \sum_{\substack{r=1 \\ p \nmid r}}^{\left\lfloor p^{l} / e\right\rfloor} \frac{1}{r^{2}} \equiv 0\left(\bmod p^{2 l}\right),
$$

and if $t=e-1$ we have

$$
\begin{aligned}
& p^{l} \sum_{\substack{r=1 \\
p \nmid r}}^{\left\lfloor(e-1) p^{l} / e\right\rfloor} \frac{1}{r^{2}} \equiv-p^{l} \sum_{\substack{r=\left\lfloor(e-1) p^{l} / e+1\right\rfloor \\
p \nmid r}}^{p^{l}} \frac{1}{r^{2}} \equiv-p^{l} \sum_{\substack{r=1 \\
p \nmid r}}^{\left\lfloor p^{l} / e\right\rfloor} \frac{1}{r^{2}}\left(\bmod p^{2 l}\right),
\end{aligned}
$$

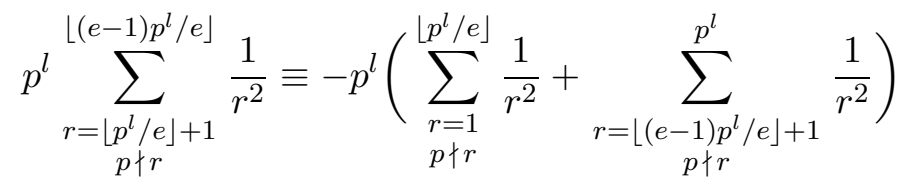

$$
\begin{aligned}
& \equiv-2 p^{l} \sum_{\substack{r=1 \\
p \nmid r}}^{\left\lfloor p^{l} / e\right\rfloor} \frac{1}{r^{2}}\left(\bmod p^{2 l}\right) \\
& \sum_{\substack{r=\left\lfloor p^{l} / e\right\rfloor+1 \\
p \nmid r}}^{p^{l}} \frac{1}{r}+\sum_{\substack{r=1 \\
p \nmid r}}^{\left\lfloor(e-1) p^{l} / e\right\rfloor} \frac{1}{r+d_{0} p^{l}} \equiv \sum_{\substack{r=1 \\
p \nmid r}}^{\left\lfloor(e-1) p^{l} / e\right\rfloor}\left(\frac{1}{p^{l}-r}+\frac{1}{r+d_{0} p^{l}}\right) \\
& \equiv-\left(1+d_{0}\right) p^{l} \sum_{\substack{r=1 \\
p \nmid r}}^{\left\lfloor(e-1) p^{l} / e\right\rfloor} \frac{1}{r^{2}} \equiv\left(1+d_{0}\right) p^{l} \sum_{\substack{r=1 \\
p \nmid r}}^{\left\lfloor p^{l} / e\right\rfloor} \frac{1}{r^{2}}\left(\bmod p^{2 l}\right),
\end{aligned}
$$

therefore,

$$
D \equiv\left\{\frac{d-1}{e^{2}}+\frac{2}{e^{2}}-\frac{1}{e}\left(1+d_{0}\right)\right\} p^{l} \sum_{\substack{r=1 \\ p \nmid r}}^{\left\lfloor p^{l} / e\right\rfloor} \frac{1}{r^{2}} \equiv 0\left(\bmod p^{2 l}\right)
$$

Lemma 4. Let $e=2,3,4$ or $6, n$ be odd, $3 \nmid n$ for $e=3$ or 6 . Then

$$
(-1)^{\phi_{e}(n)}=-1
$$

only when $n$ is a prime power $p^{\alpha}$ with $p \equiv 3(\bmod 4)$ for $e=2, p \equiv 2$ $(\bmod 3)$ for $e=3, p \equiv 3$ or $5(\bmod 8)$ for $e=4$, and $p \equiv 7$ or $11(\bmod 12)$ for $e=6$.

Proof. Let $p_{1}^{\alpha_{1}} \cdots p_{s}^{\alpha_{s}}$ be the standard factorization of $n$. If $e=2$, we assume that $p_{i} \equiv t_{i}(\bmod 4), t_{i}= \pm 1$. Then 


$$
\begin{aligned}
\phi_{2}(n) & \equiv \sum_{\substack{\alpha_{i}-1 \leq \beta_{i} \leq \alpha_{i} \\
1 \leq i \leq s}}\left\lfloor\frac{p_{1}^{\beta_{1}} \cdots p_{s}^{\beta_{s}}}{2}\right\rfloor \equiv \sum_{\substack{\alpha_{i}-1 \leq \beta_{i} \leq \alpha_{i} \\
1 \leq i \leq s}} \frac{1-t_{1}^{\beta_{1}} \cdots t_{s}^{\beta_{s}}}{2} \\
& \equiv \sum_{\substack{\alpha_{i}-1 \leq \beta_{i} \leq \alpha_{i} \\
1 \leq i \leq s}}\left\{\frac{1-t_{1}^{\beta_{1}}}{2}+\cdots+\frac{1-t_{s}^{\beta_{s}}}{2}\right\}(\bmod 2) .
\end{aligned}
$$

Since for any $1 \leq j \leq s$,

$$
\begin{aligned}
\sum_{\substack{\alpha_{i}-1 \leq \beta_{i} \leq \alpha_{i} \\
1 \leq i \leq s}} \frac{1-t_{j}^{\beta_{j}}}{2} & =\left(\sum_{\substack{\alpha_{i}-1 \leq \beta_{i} \leq \alpha_{i} \\
1 \leq i \leq s, i \neq j}} 1\right) \cdot \sum_{\alpha_{j}-1 \leq \beta_{j} \leq \alpha_{j}} \frac{1-t_{j}^{\beta_{j}}}{2} \\
& =2^{s-1} \sum_{\alpha_{j}-1 \leq \beta_{j} \leq \alpha_{j}} \frac{1-t_{j}^{\beta_{j}}}{2}
\end{aligned}
$$

we have

$$
\begin{aligned}
\phi_{2}(n) & \equiv 2^{s-1}\left\{\sum_{\alpha_{1}-1 \leq \beta_{1} \leq \alpha_{1}} \frac{1-t_{1}^{\beta_{1}}}{2}+\cdots+\sum_{\alpha_{s}-1 \leq \beta_{s} \leq \alpha_{s}} \frac{1-t_{s}^{\beta_{s}}}{2}\right\} \\
& \equiv 2^{s-1} \sum_{\substack{1 \leq i \leq s \\
t_{i}=-1}} 1(\bmod 2),
\end{aligned}
$$

where we use the fact that for any odd $a_{1}, \ldots, a_{s}$,

$$
\frac{1-a_{1} \cdots a_{s}}{2} \equiv \frac{1-a_{1}}{2}+\cdots+\frac{1-a_{s}}{2}(\bmod 2),
$$

and $\phi_{2}(n)$ is odd only when $n$ is a prime power $p^{l}$ with $p \equiv 3(\bmod 4)$.

If $e=3$, we assume that $p_{i} \equiv t_{i}(\bmod 6), t_{i}= \pm 1$. Then

$$
\begin{aligned}
& \phi_{3}(n) \equiv \sum_{\substack{\alpha_{i}-1 \leq \beta_{i} \leq \alpha_{i} \\
1 \leq i \leq s}}\left\lfloor\frac{p_{1}^{\beta_{1}} \cdots p_{s}^{\beta_{s}}}{3}\right\rfloor \equiv \sum_{\substack{\alpha_{i}-1 \leq \beta_{i} \leq \alpha_{i} \\
1 \leq i \leq s}} \frac{1-t_{1}^{\beta_{1}} \cdots t_{s}^{\beta_{s}}}{2} \\
& \equiv \sum_{\substack{\alpha_{i}-1 \leq \beta_{i} \leq \alpha_{i} \\
1 \leq i \leq s}}\left\{\frac{1-t_{1}^{\beta_{1}}}{2}+\cdots+\frac{1-t_{s}^{\beta_{s}}}{2}\right\} \\
& \equiv 2^{s-1}\left\{\sum_{\alpha_{1}-1 \leq \beta_{1} \leq \alpha_{1}} \frac{1-t_{1}^{\beta_{1}}}{2}+\cdots+\sum_{\alpha_{s}-1 \leq \beta_{s} \leq \alpha_{s}} \frac{1-t_{s}^{\beta_{s}}}{2}\right\} \\
& \equiv 2^{s-1} \sum_{\substack{1 \leq i \leq s \\
t_{i}=-1}} 1(\bmod 2)
\end{aligned}
$$

and $\phi_{3}(n)$ is odd only when $n$ is a prime power $p^{l}$ with $p \equiv 2(\bmod 3)$. 
If $e=4$, we assume that $p_{i} \equiv t_{i}(\bmod 8), t_{i}= \pm 1$ or \pm 3 . Then

$$
\begin{aligned}
\phi_{4}(n) & \left.\equiv \sum_{\substack{\alpha_{i}-1 \leq \beta_{i} \leq \alpha_{i} \\
1 \leq i \leq s}} \mid \frac{p_{1}^{\beta_{1}} \cdots p_{s}^{\beta_{s}}}{4}\right\rfloor \\
& \equiv \sum_{\substack{\alpha_{i}-1 \leq \beta_{i} \leq \alpha_{i} \\
1 \leq i \leq s}} \frac{\left(1-t_{1}^{\beta_{1}} \cdots t_{s}^{\beta_{s}}\right)\left(3-t_{1}^{\beta_{1}} \cdots t_{s}^{\beta_{s}}\right)}{8} \\
& \equiv \sum_{\substack{\alpha_{i}-1 \leq \beta_{i} \leq \alpha_{i} \\
1 \leq i \leq s}}\left\{\frac{\left(1-t_{1}^{\beta_{1}}\right)\left(3-t_{1}^{\beta_{1}}\right)}{8}+\cdots+\frac{\left(1-t_{s}^{\beta_{s}}\right)\left(3-t_{s}^{\beta_{s}}\right)}{8}\right\} \\
& \equiv 2^{s-1} \sum_{\substack{1 \leq i \leq s \\
\sum_{i}-1 \leq \beta_{i} \leq \alpha_{i}}} \frac{\left(1-t_{i}^{\beta_{i}}\right)\left(3-t_{i}^{\beta_{i}}\right)}{8} \equiv 2^{s-1} \sum_{\substack{1 \leq i \leq s \\
t_{i}=-1 \text { or }-3}} 1(\bmod 2),
\end{aligned}
$$

where we use the fact that for any odd $a_{1}, \ldots, a_{s}$,

$$
\frac{\left(1-\prod_{i=1}^{s} a_{i}\right)\left(3-\prod_{i=1}^{s} a_{i}\right)}{8} \equiv \sum_{1 \leq i \leq s} \frac{\left(1-a_{i}\right)\left(3-a_{i}\right)}{8}(\bmod 2),
$$

and $\phi_{4}(n)$ is odd only when $n$ is a prime power $p^{l}$ with $p \equiv 5$ or $7(\bmod 8)$.

If $e=6$, we assume that $p_{i} \equiv t_{i}(\bmod 12), t_{i}= \pm 1$, or \pm 5 . Then

$$
\begin{aligned}
\phi_{6}(n) & \left.\equiv \sum_{\substack{\alpha_{i}-1 \leq \beta_{i} \leq \alpha_{i} \\
1 \leq i \leq s}} \mid \frac{p_{1}^{\beta_{1}} \cdots p_{s}^{\beta_{s}}}{6}\right\rfloor \\
& \equiv \sum_{\substack{\alpha_{i}-1 \leq \beta_{i} \leq \alpha_{i} \\
1 \leq i \leq s}} \frac{\left(1-t_{1}^{\beta_{1}} \cdots t_{s}^{\beta_{s}}\right)\left(5-t_{1}^{\beta_{1}} \cdots t_{s}^{\beta_{s}}\right)}{12} \\
& \equiv \sum_{\substack{\alpha_{i}-1 \leq \beta_{i} \leq \alpha_{i} \\
1 \leq i \leq s}}\left\{\frac{\left(1-t_{1}^{\beta_{1}}\right)\left(5-t_{1}^{\beta_{1}}\right)}{12}+\cdots+\frac{\left(1-t_{s}^{\beta_{s}}\right)\left(5-t_{s}^{\beta_{s}}\right)}{12}\right\} \\
& \equiv 2^{s-1} \sum_{\substack{1 \leq i \leq s \\
\sum_{i}-1 \leq \beta_{i} \leq \alpha_{i}}} \frac{\left(1-t_{i}^{\beta_{i}}\right)\left(5-t_{i}^{\beta_{i}}\right)}{12} \equiv 2^{s-1} \sum_{\substack{1 \leq i \leq s \\
t_{i}=-1 \text { or }-5}} 1(\bmod 2),
\end{aligned}
$$

where we use the fact that for any $a_{i} \equiv \pm 1$ or $\pm 5(\bmod 12), 1 \leq i \leq s$,

$$
\frac{\left(1-\prod_{i=1}^{s} a_{i}\right)\left(5-\prod_{i=1}^{s} a_{i}\right)}{12} \equiv \sum_{1 \leq i \leq s} \frac{\left(1-a_{i}\right)\left(5-a_{i}\right)}{12}(\bmod 2),
$$

and $\phi_{6}(n)$ is odd only when $n$ is a prime power $p^{l}$ with $p \equiv 7$ or $11(\bmod 12)$. This completes the proof of Lemma 4. 


\section{Proof of Theorem 1}

Proof of (2). We first assume that $n=p^{l}$ with prime $p \geq 5$. Taking $t=2$ and $2 k=\phi\left(p^{2 l}\right)$ in (9), by using Lemma 2 with $e=2$ and (11), we have

$$
\begin{aligned}
\sum_{\substack{r=1 \\
p \nmid r}}^{\left(p^{l}-1\right) / 2} \frac{1}{p^{l}-2 r} & \equiv \sum_{r=1}^{\left(p^{l}-1\right) / 2}\left(p^{l}-2 r\right)^{\phi\left(p^{2 l}\right)-1} \\
& \equiv q_{2}\left(p^{2 l}\right) \equiv q_{2}\left(p^{l}\right)-\frac{1}{2} q_{2}^{2}\left(p^{l}\right) p^{l}\left(\bmod p^{2 l}\right) .
\end{aligned}
$$

Now, let $n=p^{l} m, p \geq 3, m>1, p \nmid m$. By Lemma 3 and (16),

$$
\begin{aligned}
\sum_{\substack{r=1 \\
(r, n)=1}}^{(n-1) / 2} \frac{1}{n-2 r} \equiv & \sum_{\substack{r=1 \\
p \nmid r}}^{(n-1) / 2} \frac{1}{n-2 r}-\sum_{\substack{r=1 \\
p \nmid r \\
q_{1} \mid r}}^{(n-1) / 2} \frac{1}{n-2 r} \\
& +\cdots+(-1)^{s} \sum_{\substack{r=1 \\
p \nmid r \\
q_{1} \cdots q_{g} \mid r}}^{\substack{q_{1} \cdots / 2 \\
n-2 r}} \\
\equiv & \prod_{q \mid m}\left(1-\frac{1}{q}\right) \sum_{\substack{r=1 \\
p \nmid r}}^{\left(p^{l}-1\right) / 2} \frac{1}{p^{l}-2 r} \\
\equiv & \frac{\phi(m)}{m}\left(q_{2}\left(p^{l}\right)-\frac{1}{2} p^{l} q_{2}^{2}\left(p^{l}\right)\right)\left(\bmod p^{2 l}\right),
\end{aligned}
$$

where $q_{i}(1 \leq i \leq g)$ are the prime factors of $m$.

On the other hand,

$$
\begin{aligned}
q_{2}(n) & =\frac{\left(1+p^{l} q_{2}\left(p^{l}\right)\right)^{\phi(m)}-1}{p^{l} m} \\
& \equiv \frac{\phi(m)}{m} q_{2}\left(p^{l}\right)+\frac{\phi(m)(\phi(m)-1)}{2 m} p^{l} q_{2}^{2}\left(p^{l}\right)\left(\bmod p^{2 l}\right), \\
n q_{2}^{2}(n) & \equiv n\left(\frac{\phi(m)}{m} q_{2}\left(p^{l}\right)\right)^{2} \equiv \frac{\phi^{2}(m)}{m} p^{l} q_{2}^{2}\left(p^{l}\right)\left(\bmod p^{2 l}\right),
\end{aligned}
$$

hence

$$
q_{2}(n)-\frac{1}{2} n q_{2}^{2}(n) \equiv \frac{\phi(m)}{m}\left(q_{2}\left(p^{l}\right)-\frac{1}{2} p^{l} q_{2}^{2}\left(p^{l}\right)\right)\left(\bmod p^{2 l}\right) .
$$

Combining (17) with (18) yields (2).

Proof of (3). Similarly, we first assume that $n=p^{l}$ with prime $p \geq 5$. Taking $t=3$ and $2 k=\phi\left(p^{2 l}\right)$ in (9), by using Lemma 2 with $e=3$ and (11), 
we have

$$
\begin{aligned}
\sum_{\substack{r=1 \\
p \nmid r}}^{\left\lfloor\left(p^{l}-1\right) / 3\right\rfloor} \frac{1}{p^{l}-3 r} & \equiv \sum_{r=1}^{\left\lfloor\left(p^{l}-1\right) / 3\right\rfloor}\left(p^{l}-3 r\right)^{\phi\left(p^{2 l}\right)-1} \\
& \equiv \frac{1}{2} q_{3}\left(p^{2 l}\right) \equiv \frac{1}{2} q_{3}\left(p^{l}\right)-\frac{1}{4} q_{3}^{2}\left(p^{l}\right) p^{l}\left(\bmod p^{2 l}\right) .
\end{aligned}
$$

If $n=p^{l} m, p \geq 5, m>1, p \nmid m, 3 \nmid m$, noting that $m \equiv \pm 1(\bmod 6)$, by using Lemma 3 and (19), we can prove (3) in a way similar to (2).

Proof of (4). Taking $t=4$ and $2 k=\phi\left(p^{2 l}\right)$ in (9), by using Lemma 2 with $e=4$ and (11), we have

$$
\begin{aligned}
& \sum_{\substack{r=1 \\
p \nmid r}}^{\left\lfloor\left(p^{l}-1\right) / 4\right\rfloor} \frac{1}{p^{l}-4 r} \equiv \sum_{r=1}^{\left\lfloor\left(p^{l}-1\right) / 4\right\rfloor}\left(p^{l}-4 r\right)^{\phi\left(p^{2 l}\right)-1} \\
& \quad \equiv \frac{1}{4}\left(q_{4}\left(p^{2 l}\right)+q_{2}\left(p^{2 l}\right)\right) \equiv \frac{3}{4} q_{2}\left(p^{l}\right)-\frac{3}{8} q_{2}^{2}\left(p^{l}\right) p^{l}\left(\bmod p^{2 l}\right) .
\end{aligned}
$$

If $n=p^{l} m, p \geq 5, m>1, p \nmid m$, noting that $m \equiv \pm 1(\bmod 4)$, by using Lemma 3 and (20), we can prove (4) in a way similar to (2).

Proof of (5). Taking $t=6$ and $2 k=\phi\left(p^{2 l}\right)$ in (9), by using Lemma 2 with $e=6$ and (11), we have

$$
\begin{aligned}
\sum_{\substack{r=1 \\
p \nmid r}}^{\left\lfloor\left(p^{l}-1\right) / 6\right\rfloor} & \frac{1}{p^{l}-6 r} \equiv \sum_{r=1}^{\left\lfloor\left(p^{l}-1\right) / 6\right\rfloor}\left(p^{l}-6 r\right)^{\phi\left(p^{2 l}\right)-1} \\
& \equiv \frac{1}{12}\left(q_{6}\left(p^{2 l}\right)+2 q_{3}\left(p^{2 l}\right)+3 q_{2}\left(p^{2 l}\right)\right) \\
& \equiv \frac{1}{3} q_{2}\left(p^{l}\right)+\frac{1}{4} q_{3}\left(p^{l}\right)-\frac{1}{6} p^{l} q_{2}^{2}\left(p^{l}\right)-\frac{1}{8} p^{l} q_{3}^{2}\left(p^{l}\right)\left(\bmod p^{2 l}\right) .
\end{aligned}
$$

If $n=p^{l} m, p \geq 7, m>1, p \nmid m$, noting that $2 \nmid n, 3 \nmid n, m \equiv \pm 1(\bmod 6)$, by using Lemma 3 and (21), we can prove (5) once again as above.

4. Proof of Theorem 2. Define

$$
A_{n}=\left(\begin{array}{l}
n-1 \\
\lfloor n / e\rfloor
\end{array}\right) \text {. }
$$

Then

$$
A_{n}=\prod_{r=1}^{\lfloor n / e\rfloor} \frac{n-r}{r}=\prod_{d \mid n} \prod_{\substack{r=1 \\(r, n)=d}}^{\lfloor n / e\rfloor} \frac{n-r}{r}=\prod_{d \mid n} T_{n / d}=\prod_{d \mid n} T_{d}
$$


where

$$
T_{d}=\prod_{\substack{r=1 \\(r, d)=1}}^{\lfloor d / e\rfloor} \frac{d-r}{r}
$$

Using the Möbius inversion formula, we have

$$
T_{n}=\prod_{d \mid n} A_{d}^{\mu(n / d)}=\prod_{d \mid n}\left(\begin{array}{l}
d-1 \\
\lfloor/ e\rfloor
\end{array}\right)^{\mu(n / d)} .
$$

On the other hand,

$$
\begin{aligned}
& T_{n}=\prod_{\substack{r=1 \\
(r, n)=1}}^{\lfloor n / e\rfloor} \frac{n-r}{r}=(-1)^{\phi_{e}(n)} \prod_{\substack{r=1 \\
(r, n)=1}}^{\lfloor n / e\rfloor}\left(1-\frac{n}{r}\right) \\
& \equiv(-1)^{\phi_{e}(n)}\left\{1-n \sum_{\substack{r=1 \\
(r, n)=1}}^{\lfloor n / e\rfloor} \frac{1}{r}\right\} \\
& \equiv(-1)^{\phi_{e}(n)}\left\{1+e n \sum_{\substack{r=1 \\
(r, n)=1}}^{\lfloor n / e\rfloor} \frac{1}{n-e r}\right\}\left(\bmod n^{2}\right) \text {, }
\end{aligned}
$$

where

$$
\phi_{e}(n)=\sum_{\substack{r=1 \\(r, n)=1}}^{\lfloor n / e\rfloor} 1=\sum_{r=1}^{\lfloor n / e\rfloor} \sum_{d \mid(r, n)} \mu(d)=\sum_{d \mid n} \mu(d)\left\lfloor\frac{n}{d e}\right\rfloor=\sum_{d \mid n} \mu\left(\frac{n}{d}\right)\left\lfloor\frac{d}{e}\right\rfloor,
$$

and applying Lemma 4 and (2)-(4) to (22), respectively, we complete the proof of Theorem 2.

If we set $d=p q$ in Theorem 2 , then Corollary 1 can be derived easily with the help of Lemma 4.

5. Proof of Theorem 3. Taking $t=4$ and $2 k=\phi\left(p^{l}\right)-2$ in (10), and using the von Staudt-Clausen theorem, we have

$$
\sum_{\substack{r=1 \\ p \nmid r}}^{\left\lfloor p^{l} / 4\right\rfloor} \frac{1}{r^{2}} \equiv \sum_{r=1}^{\left\lfloor p^{l} / 4\right\rfloor} r^{\phi\left(p^{l}\right)-2} \equiv-\frac{B_{\phi\left(p^{l}\right)-1}(s / 4)}{\phi\left(p^{l}\right)-1} \begin{cases}\left(\bmod p^{l}\right) & \text { for } p \geq 5, \\ \left(\bmod 3^{l-1}\right) & \text { for } p=3,\end{cases}
$$

where we have written $s$ for the least positive residue of $p^{l}$ modulo 4 . By using Lemma 2, we have 


$$
B_{\phi\left(p^{l}\right)-1}\left(\frac{s}{4}\right)=-(-1)^{\left(p^{l}-1\right) / 2} \frac{\left(\phi\left(p^{l}\right)-1\right) E_{\phi\left(p^{l}\right)-2}}{4^{\phi\left(p^{l}\right)-1}} .
$$

Applying (23) to (24) yields

$$
\sum_{\substack{r=1 \\ p \nmid r}}^{\left\lfloor p^{l} / 4\right\rfloor} \frac{1}{r^{2}} \equiv(-1)^{\left(p^{l}-1\right) / 2} 4 E_{\phi\left(p^{l}\right)-2} \begin{cases}\left(\bmod p^{l}\right) & \text { for } p \geq 5, \\ \left(\bmod 3^{l-1}\right) & \text { for } p=3,\end{cases}
$$

which completes the proof of Theorem 3 .

Acknowledgements. The authors are grateful to the referee for his valuable suggestions and constructive report which made this paper more readable.

\section{References}

[1] T. X. Cai, A congruence involving the quotients of Euler and its applications (I), Acta Arith. 103 (2002), 313-320.

[2] E. Lehmer, On congruences involving Bernoulli numbers and the quotients of Fermat and Wilson, Ann. of Math. 39 (1938), 350-359.

[3] P. Ribenboim, 13 Lectures on Fermat's Last Theorem, Springer, 1979.

Department of Mathematics

Zhejiang University

Hangzhou, 310027, P.R. China

E-mail: txcai@mail.hz.zj.cn

fuxudan@126.com

xiazhou0821@hotmail.com

Received on 3.5.2006

and in revised form on 4.4.2007 